\title{
The PIG in sPrInG: Evidence on letter grouping from the reading of buried words
}

\author{
GLYN W. HUMPHREYS \\ University of Birmingham, Birmingham, England \\ KATE MAYALL \\ University of Leicester, Leicester, England \\ and \\ ADAM C. G. COOPER \\ University of Birmingham, Birmingham, England
}

\begin{abstract}
We introduce a novel procedure for investigating factors that determine selective attention to letters in words. Participants were presented with words (in Experiments 1 and 3) and nonwords (in Experiment 2) that contained a buried word whose letters differed in color relative to the other letters present (e.g., pig, in spring). The strings were presented in single case or mixed case, keeping the letters of the buried words in one case $(\mathrm{S} p \mathrm{R} i \mathrm{Ng})$. The time in which the whole stimulus was named was shorter for same-case than for mixed-case strings (for spring: spring $<\mathrm{S} p \mathrm{R} i \mathrm{~N} g$ ). In contrast, the time in which buried words were named was shorter in mixed- than in same-case strings (for pig: spring > $\mathrm{S} p \mathrm{R} i \mathrm{~N} g$ ). Across items, the effects of case mixing were negatively correlated across the two tasks. The positive effect of case mixing for buried words also occurred irrespective of whether the whole string was a word or a nonword, and there were contributions from similarity of both letter size and case. The results suggest that case mixing can facilitate selective attention to letters, which is otherwise disrupted by size- and case-based grouping across letter strings. The study provides evidence for letter grouping using size and case information.
\end{abstract}

There is considerable evidence that skilled readers process the letters in words in a spatially parallel manner, provided that the words are no longer than about six letters long (e.g., Carr \& Pollatsek, 1985). For example, both lexical decision times and word-naming times are unaffected by increases in word length from four to six letters (Frederiksen \& Kroll, 1976; Weekes, 1997), and, when four-letter words are read, attention seems to be distributed evenly across the string (e.g., as measured with a dot detection procedure; LaBerge, 1983). Parallel processing within words can allow the letters to group together. Some evidence for letter grouping comes from the study of the effects of case mixing on word reading. It has long been known that mixed-case words are more difficult to name than words presented in single case (either upper or lower; see Coltheart \& Freeman, 1974; Smith, 1969). Mayall, Humphreys, and Olson (1997) found that the case-mixing effect was reduced when

This work was supported by grants from the BBSRC and the Wellcome Trust (U.K.). Correspondence concerning this article should be addressed to G. W. Humphreys, School of Psychology, University of Birmingham, Edgbaston, Birmingham B15 2TT, England (e-mail: g.w.humphreys@bham.ac.uk).

Note-This article was accepted by the previous editorial team, while John T. Wixted was editor. spaces were interspersed between the letters; naming times to mixed-case words were selectively speeded. Mayall et al. proposed that spacing disrupted grouping between letters in the same case. With mixed-case strings, case-based grouping would disrupt word identification, because inappropriate units would address the lexicon (e.g., in a stimulus such as $\mathrm{SpRiNg}$, case-based grouping would yield the units pig and $S R N$, neither of which corresponds to the lexical entry for spring).

Yet, despite over 100 years of research into reading (e.g., Cattell, 1886), few researchers have asked how the parallel processing of letters in words, and any consequent grouping, impacts on tasks that require selective attention to a subset of the letters present. Many experiments have been conducted into single-letter identification in words, typically using the Reicher-Wheeler paradigm (Reicher, 1969; Wheeler, 1970). These studies have shown that identification is benefitted when a letter is part of a word, relative to when it is part of a nonword - the word superiority effect (WSE) — a result that can be modeled in terms of feedback from lexical knowledge to letter-level processing (McClelland \& Rumelhart, 1981). However, although this paradigm requires attention to letters within words and nonwords, it is not necessarily informative about the influence of letter groups on attention within the string. For example, it might be that familiar letter groups disrupt selective at- 
tention within words but that this is hidden by effects of lexical feedback, leading to the WSE. It may also be that letter grouping is influenced by such factors as letter size and case, which are matched in words and nonwords, and so the contrast between these stimuli is insensitive to the factors that modulate letter grouping and its effect on selective attention.

In the present study, we introduce a new paradigm designed to investigate how grouping between letters affects selective attention: the buried-word procedure. Participants were presented with six-letter words or nonwords, with alternate letters in red and green. In each string, three of the letters (shown in red) formed a word in their own right; this is the buried word. In Experiment 1, the participants had to name either the whole strings (in one trial block) or the buried words (in another trial block). In Experiments 2 and 3, they named only the buried words. The strings were also shown with same-case letters or with alternate letters in different cases (as well as colors). Examples are spring (same case) and $\mathrm{S} p \mathrm{R} i \mathrm{Ng}$ (mixed case). In cases in which the participants named the whole string, we would expect same-case strings to be responded to more rapidly than mixed-case strings: There should be a negative effect of case mixing (as has been shown on numerous previous occasions; see Besner \& Johnston, 1989; Besner \& McCann, 1987). Now consider performance when the participants named buried words, a task that required selective attention to components of the whole string. If there was grouping between the letters of the same case and size across the strings, it might be more difficult to read buried words in singlecase strings (e.g., spring) than in strings with mixed-case letters (e.g., $\mathrm{S} p \mathrm{R} i \mathrm{Ng}$ ), since grouping between the buried word and the other letters would be stronger in the samecase stimuli. ${ }^{1}$ This would be consistent with findings in the object perception literature in which grouping disrupts attention to the components of a stimulus (e.g., Rensink \& Enns, 1995). In the present context, this would generate a novel result in which performance would be better with mixed-case than with single-case stimuli. Clear evidence for this was shown in Experiment 1. Furthermore, the size of the case-mixing impairment was correlated with the size of the case-mixing advantage across items. Apparently, strong grouping between same-case letters speeds naming of the whole word but impedes selective attention to the letters, which are needed to read the buried words. This suggests that the buried-word paradigm can inform us not only about how attention is allocated to letters in words, but also about the supraletter units that contribute to reading at the whole-word level (since these units disrupt buried-word reading).

In Experiments 2 and 3, we examined the factors that lead to this mixed-case advantage in the buried-word task. Experiment 2 contrasted the reading of buried words in strings that were words or nonwords, to assess whether familiarity at the whole-string level would disrupt the naming of buried words when all the same let- ters had the same size and case. We did not find evidence for a lexical contribution. In Experiment 3, we evaluated whether there would be grouping based on case or size, by varying these factors independently (rather than these factors being varied jointly, as in "standard" MiXeD case). We present new evidence that similarity of case, as well as size, is important. We discuss the evidence in relation to the formation of letter groups when both buried words and whole words are read.

\section{EXPERIMENT 1 The Buried-Word Effect}

\section{Method}

Stimuli and Apparatus. The stimuli consisted of 120 six-letter words, each of which contained a buried three-letter word from either the odd- or the even-numbered letters present (beauty $\rightarrow$ bat; spring $\rightarrow$ pig). There were 60 strings in which the buried words were formed from the odd letters and 60 in which the buried words were formed from the even letters. The letters forming the buried word appeared in red, and the other letters making up the whole string appeared in green. All the letters were also either in the same case (either upper or lower) or in mixed cases. With mixed-case strings, the letters in the buried word were always in the same case; half of the time, this was uppercase $(\operatorname{s} \operatorname{Pr} I n G)$, and in the other half, it was lowercase $(\mathrm{S} p \mathrm{R} i \mathrm{Ng}$ ). The stimuli appeared in size 12 Geneva font, at the center of the screen of a Pentium PC.

Participants. There were 24 participants, all from the University of Birmingham. Each had normal or corrected-to-normal vision and was between 18 and 23 years of age.

Design and Procedure. On each trial, the participants received a central white fixation cross against a black background for $500 \mathrm{msec}$, followed by a six-letter word (which was exposed until the participants responded). There were two blocks of trials. In one block, the participants named the complete word; in the other, they named the buried word (presented in red). The order of these two blocks was counterbalanced over participants. Naming responses triggered a voice key, and responses were timed by the computer.

The word set was divided into eight groups, defined by the task (read whole or buried word) and by the case of the letters (e.g., spring, $\mathrm{S} P \mathrm{R} I \mathrm{~N} G, \mathrm{~S} p \mathrm{R} i \mathrm{~N} g$, and $\mathrm{s} P \mathrm{r} I \mathrm{n} G)$. There were 15 words per group, which were rotated across eight subgroups of participants (3 per group) so that no participant saw a word more than once. The order of the words within a block was randomized for each participant.

\section{Results}

Of the trials, $2.8 \%$ were excluded due to voice key errors. Owing to a computer error, data from 1 participant were also lost. Preliminary analyses of variance (ANOVAs) on all the remaining correct response times (RTs) failed to reveal any effect of task order or whether the buried word occupied the even or the odd positions within the whole string (all $F_{\mathrm{S}}<1$ ). However, there was a reliable main effect of task [read whole word vs. buried word; $F_{1}(1,22)=109.06$ and $F_{2}(1,117)=590.67$; both $p$ s $<$ $.001] .{ }^{2}$ Whole words were read more quickly than buried words (622 vs. $922 \mathrm{msec}$ ). Neither the main effect of case mixing nor that of buried-word case approached significance (all $F_{\mathrm{S}}<1$ ). There was one reliable interaction, between task and case mixing $\left[F_{1}(1,22)=64.53\right.$ and $F_{2}(1,117)=95.63$; both $\left.p \mathrm{~s}<.001\right]$. This interaction is shown in Figure 1, which presents the mean correct 


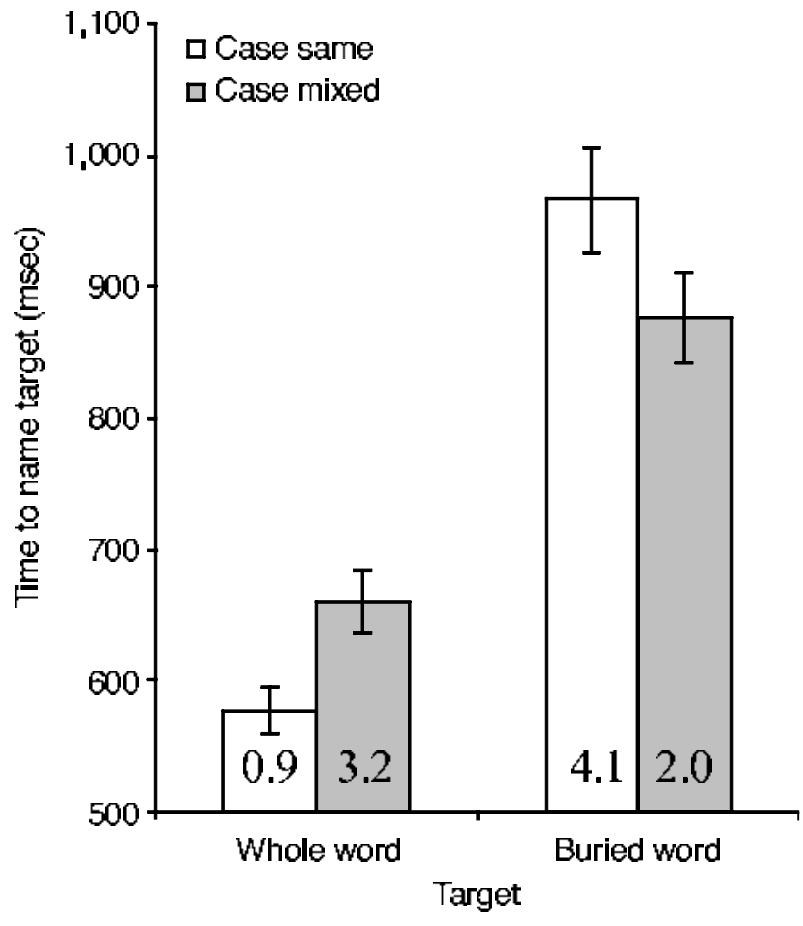

Figure 1. Mean correct response times (in milliseconds) as a function of the reading task (whole string or buried word) and the case of the whole string (same case or mixed case) in Experiment 1 . Error bars indicate $95 \%$ confidence intervals. The percentage of errors for each condition is given as a number.

RTs (in milliseconds), averaged over whether the target stimulus (whole or buried word) was upper- or lowercase. The case of the buried word not only was unreliable as a main effect, but also failed to interact with either of the other factors.

Taking whole-word naming responses alone, there was a reliable effect of case mixing: Same-case words were named more quickly than different-case words [a negative case-mixing effect of $74 \mathrm{msec} ; F_{1}(1,23)=20.82$, $p<.001]$. Taking naming responses to buried words alone, stimuli in mixed-case strings were named more quickly than those in same-case strings [a positive casemixing effect of $\left.111 \mathrm{msec} ; F_{1}(1,23)=186.94, p<.001\right]$.

We also examined the relations between the size of the case-mixing effect on whole-word reading and on buriedword reading. To do this, we took for each item and for each task the average difference between RTs when critical letters were presented in a single-case string (spring) and when they appeared in a mixed-case string $(\mathrm{S} p \mathrm{R} i \mathrm{Ng})$. Across items, there were reliable negative correlations between the effects of case mixing in the two tasks [for critical lowercase items, $r(118)=-.37$, and for critical uppercase items, $r(118)=-.30$; both $p$ s $<.001]$. Stimuli that were most disrupted by case mixing in the wholeword reading task benefitted the most from case mixing in the buried-word task.

The error rates were generally low and followed the same trends as those for the RTs. Overall, more errors were made in the buried-word task than the whole-word reading task. In the whole-word task, more errors were made on mixed-case than on same-case stimuli, whereas the opposite occurred in the buried-word reading task. There was no evidence of a speed-accuracy tradeoff.

\section{Discussion}

The results were clear. As has been found on numerous other occasions, whole-word naming responses were slower to mixed-case than to same-case letter strings (for representative data, see Besner \& Johnston, 1989; Besner \& McCann, 1987; Mayall \& Humphreys, 1996; Mayall et al., 1997). The task of naming the buried word was also more difficult than that of naming the whole words (RTs were longer, and errors increased). This is not surprising given that reading a subpart of a letter string is a relatively unpracticed task and one that requires selective attention within the letter string. What is more interesting is that the effects of case mixing were exactly the opposite for the buried-word task and the wholeword reading task. This is consistent with grouping's disrupting selective attention to the letters composing the buried words within the strings. Grouping between letters of the same case and size (in same-case strings) facilitates reading across whole words but impairs attention to groups of letters, a requirement for the reading of buried words. Case mixing disrupts letter grouping. As a consequence, whole-word reading is slowed, whereas in contrast, buried words are attended to and named more rapidly. On this argument, similar letter-grouping processes come into play in the reading of buried words and of whole words. This is supported by the correlation between the size of the cost due to case mixing on wholeword reading and the size of the benefit of case mixing on the reading of buried words. The items most susceptible to the negative effects of letters of the same case and size in buried words benefited the most from the same case and size letters when the task was whole-word reading, relative to when the letters were mixed in case and size.

Other accounts of these data are also possible, however. One is that the results in the buried-word task reflected response interference from the whole word. When required to name the buried word, participants may encounter competition between the name of the buried word and that of the whole, parent word that it is a part of. Such competition will slow naming. Now, in studies of response competition in the Stroop colorword task, it is well known that interference from an irrelevant color word is dependent, in part, on the relative speed with which responses can be formed to the relevant and the irrelevant properties of stimuli (MacLeod, 1992). Interference effects are reduced when responses to the irrelevant properties are slowed. The whole-word naming task demonstrates that responses to the whole word are slowed for mixed-relative to same-case strings. It follows that interference on a response to the buried word from a competing whole-word response will be less for mixed-case strings; as a consequence, buried words may be read relatively more quickly. 
Another account is that case mixing facilitates the segmentation of target from nontarget letters in the buriedword task not because it disrupts same-case letter groups, but because it introduces size differences between the letters. Size differences between target and nontarget letters enable buried words to be attended more easily, facilitating their identification. This may be an effect on early visual processing that is prior to the stimuli's accessing stored letter and lexical representations.

These alternative accounts were assessed in Experiments 2 and 3. In Experiment 2, we had the participants name buried words that were part of six-letter nonwords. The nonwords were formed by randomly interchanging the locations of the nontarget (green) letters from the strings used in Experiment 1, keeping constant the positions of the letters forming the buried words. The whole strings were, thus, difficult to pronounce. Response competition from the identity of the whole string should be reduced when this string is a nonword relative to when it is a word (as in Experiment 1). On the other hand, effects of inappropriate grouping between the target and the nontarget letters may still occur irrespective of whether the parent string has a stored lexical representation. There may be slowed naming of buried words in same-case relative to mixed-case strings, both when the whole strings are words and when they are nonwords.

In Experiment 3, we tested the effects of segmentation by size. The participants named buried words presented in the context of small lowercase letters. The letters making up the buried words were in lower- or uppercase, and they were either the same size as the contextual letters (both small) or smaller than the contextual letters. If segmentation by size is crucial, buried words should be read more quickly when they differ in size from the contextual letters irrespective of letter case. ${ }^{3}$ If grouping and segmentation by case are also important, buried words should be named more quickly when they differ in case from the contextual letters, irrespective of letter size.

\section{EXPERIMENT 2 Using Whole-String Nonwords}

\section{Method}

Unless otherwise mentioned, the method was the same as that in Experiment 1 . There were again 24 participants, between 18 and 24 years of age, with either normal or corrected-to-normal vision. Unlike Experiment 1, there was only one block of trials, and for this the participants were asked just to name the (red) buried words. The six-letter strings were based on those employed in the earlier experiment, except that we randomly interchanged the positions of the nontarget letters (e.g., to create $\mathrm{n} p \operatorname{sir} g$ rather than spring). This created nonwords at the level of the whole string, which were relatively difficult to pronounce. The 120 strings were divided into four groups according to the case of the letters (n $p \operatorname{sir} g, \mathrm{~N} P \mathrm{~S} I \mathrm{R} G$, $\mathrm{N} p \mathrm{SiR} g$, and $\mathrm{n} P \mathrm{~S} I \mathrm{r} G$ ), and these were counterbalanced across four groups of participants so that, for any 1 participant, no item was repeated across conditions.

\section{Results}

Of the responses, $1.5 \%$ were discarded because of voice key errors. The mean correct RTs based on the re- maining data for reading are shown in Figure 2. For comparison purposes, the data are presented along with the results from the buried-word naming task with words at the whole-string level (from Experiment 1).

To compare the magnitude of the case-mixing effect on the identification of buried words in whole-string words and nonwords, the data from Experiment 2 were analyzed in conjunction with the buried-word data from Experiment 1. Preliminary analysis again showed no evidence of an effect of whether the buried words occupied the even or the odd letter positions $(F<1)$. For the analysis across participants, there was one between-subjects factor (experiment) and two within-subjects factors (case mixing and target case). For the analysis across items, a three-factor repeated measures design was used, since the buried-word targets were the same in all instances. There was an effect of experiment across items, but not across participants $\left[F_{2}(1,117)=9.53, p<.01\right.$; $\left.F_{1}<1.0\right]$. Naming times tended to be shorter in Experiment 1 (with whole-string words) than in Experiment 2 (with whole-string nonwords). Note that this result is in the opposite direction to that which would be expected if response interference affected performance. There was also a significant effect of case mixing $\left[F_{1}(1,45)=75.01\right.$ and $F_{2}(1,117)=58.83$; both $p$ s $\left.<.001\right]$. Buried words were read more quickly in mixed-case strings than in same-case strings. The effect of target case (upper or lower) was not reliable $\left[F_{1}(1,45)=2.65, p>.05 ; F_{2}<1\right]$,

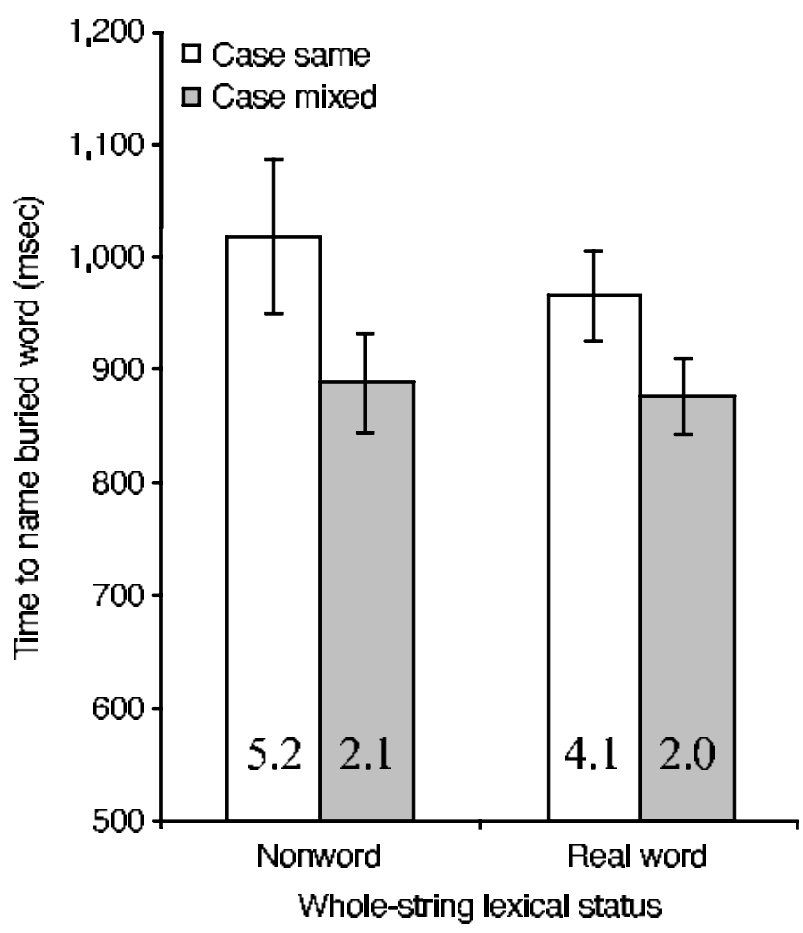

Figure 2. Buried-word reading only: mean correct response times (in milliseconds) as a function of whether the whole string was a word or a nonword and was presented in the same or in a mixed case. The data are drawn from Experiments 1 and 2. The percentages of errors are given as numbers. 
and this factor did not interact with the other variables. Importantly, the interaction between experiment (wholestring words vs. nonwords) and case mixing did not approach significance $\left[F_{1}(1,45)=2.64, p>.05 ; F_{2}<1\right]$.

The errors followed the same trends as those for RTs, with fewer errors being made to mixed- than to same-case strings. There was no indication of a speed-accuracy tradeoff.

\section{Discussion}

When the whole strings were nonwords, there remained a substantial benefit from case mixing when the participants had to read buried words. Indeed, the positive effect of case mixing was larger in Experiment 2 (whole-string nonwords) than in Experiment 1 (wholestring words; $140 \mathrm{vs.} 111 \mathrm{msec}$ ), although the interaction failed to reach significance. This result goes in the opposite direction to that which would be predicted if the positive case-mixing effect were due to reduced response interference for the buried words. Response competition from the identity of the whole strings should be weaker when the whole strings are nonwords that are difficult to pronounce, relative to when the strings are words. Despite this, the positive effects of case mixing were at least as large. In addition, overall times to name buried words tended to be longer in Experiment 2 (with whole-string nonwords) than in Experiment 1 (with whole-string words). There is no evidence for response interference on either overall RTs or the case-mixing effect. These data also suggest that the difficulty in reading buried words in single-case strings was not due to grouping based on the familiarity of the whole stimulus, since, on this account too, performance should be worse for letters buried in whole words, relative to letters buried in nonwords.

These data, indicating no effect of the lexical identity of the whole string on the reading of buried words, contrast with the effects of lexical identity on reading at the whole-string level; in many studies, it has been demonstrated that, at the whole-string level, words are named more quickly than nonwords (McCann \& Besner, 1987; Patterson \& Coltheart, 1987). This suggests that there are factors (such as lexical identity) that contribute to the reading of whole strings that do not influence selective attention to the letters forming the buried words. This is perhaps not surprising, since reading at the whole-string level is influenced by access to orthographic and phonological knowledge-processes that operate more efficiently for words than for nonwords. We suggest that these processes, sensitive to lexical identity, take place after the formation of letter groups across the string, an earlier operation that affects both buried-word and wholeword reading (but in opposite directions) and that, from the present evidence, appears to take place in the same way for letters in words and nonwords. Because of the opposite effects of letter grouping on buried-word and whole-word reading, we found that there was a negative correlation between the effects of case mixing on the two tasks (Experiment 1), despite the fact that reading at the whole-string level was affected by additional factors.

\section{EXPERIMENT 3 Effects of Size and Case}

\section{Method}

In this experiment, we compared the relative effects of case and size differences between consecutive letters on the reading of buried words. There were 12 participants, between 18 and 26 years of age, with either normal or corrected-to-normal vision. There were four conditions, all based around a task of naming a buried word presented in the context of lowercase letters (which, when combined with the letters in the buried word, made up a known word). The letters forming the buried word were the same size and case (spring), different in size but with the same case (spring), the same size but different in case $(\mathrm{S} p \mathrm{R} i \mathrm{Ng})$, or different in both size and case $(\mathrm{S} p \mathrm{R} i \mathrm{Ng})$. The critical buried words were always in lowercase, and they were always small. The distractor letters were either large or small and either upper- or lowercase. There were no effects of letter size/case per se in either Experiment 1 or Experiment 2. The large letters were the same size as the uppercase letters in Experiments 1 and 2, and the small letters were the same size as the lowercase letters in those experiments.

The 120 strings from Experiment 1 were divided into four groups, and each group was assigned to each of the four conditions, counterbalanced across participants (so that no participant saw repeats of any of the strings). The conditions were presented at random to each participant, and the task was to read the three-letter word in red on each trial.

\section{Results}

The mean correct naming times to the buried words are depicted in Figure 3, and the error rates are shown below. Of the trials, $1.7 \%$ were lost due to voice key errors. All other correct RTs were used to calculate the means for the ANOVAs.

RTs were analyzed in two two-factor ANOVAs, with repeated measures on both factors: size (letters in the buried word large or small) and case (letters in the buried word lower- or uppercase). A preliminary analysis failed to show any effect of whether the buried word occupied the odd or the even positions within the string $(F<1)$. For the analyses across participants and items, there were reliable main effects of size $\left[F_{1}(1,11)=9.82, F_{2}(1,119)=\right.$ 9.78; both $p$ s $<.01]$ and case $\left[F_{1}(1,11)=18.63\right.$ and $F_{2}(1,119)=19.89$; both $p$ s $\left.<.001\right]$. The interaction between size and case was not significant $\left[F_{1}(1,11)=1.89\right.$ and $F_{2}(1,119)=2.68$; both $p$ s $\left.>.05\right]$.

The error rate was low and followed the same pattern as that for the RTs. These data were not analyzed further.

\section{Discussion}

We found positive effects of both case and size changes between the target and the context letters on the reading of buried words. There was no interaction. These results clearly indicate that grouping by case was a contributory factor to the effects of case mixing on buriedword reading in Experiments 1 and 2, in addition to any effects based on grouping and segmentation of target and distractor letters by size. To the best of our knowledge, 


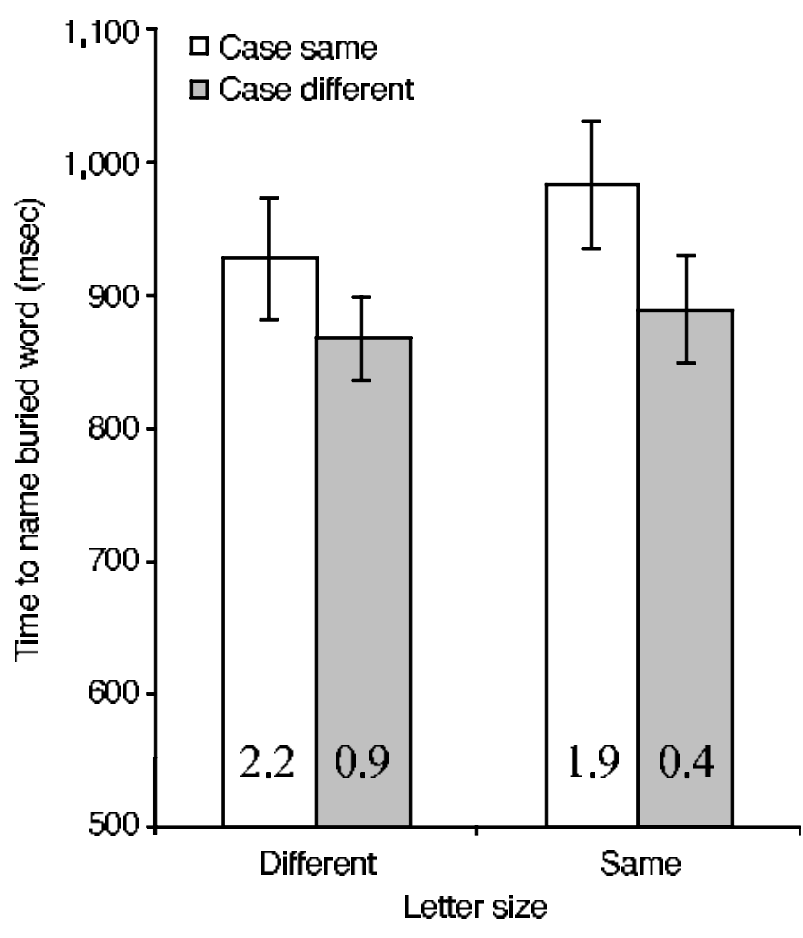

Figure 3. Buried-word reading in Experiment 3: mean correct response times (in milliseconds) as a function of whether the letters in the buried word were the same size as or a different size from the distractor letters and according to whether they were in the same case (lower) or a different case (upper). The percentages of errors are given as numbers.

this is the first result to demonstrate an effect of case on letter grouping. This case-based grouping effect may reflect the familiar co-occurrence of letters in the same case, which leads to their being encoded as a single visual unit. Encoding consecutive letters as a single visual unit is detrimental to the task of reading the buried word.

The absence of an interaction between case and size can be interpreted in terms of additive factors logic (Sternberg, 1969), with the two effects being assigned to different stages of processing. For example, similarities and differences in size may affect an initial stage of grouping/segmentation, followed by a subsequent grouping process based on co-occurrence of letters in the same case. To account for the additive pattern, however, we must assume that the first grouping/segmentation process is not all-or-none but, rather, weights letters in terms of their similarities/differences. The stronger the weight, the more likely it is that the letters activate a common unit during the subsequent recognition process (see Duncan \& Humphreys, 1989). Letters that differ in size may, thus, be less likely to activate a common representation in word recognition, but there may still be some activation if there are visual units sensitive to the co-occurrences of letter forms. As a consequence, different-sized letters in the same case will still create some activation in a common unit, disrupting the reading of the buried word. It follows that performance will be worse for same- than for different-case stimuli even when the letters contrast in size (for spring vs. $\mathrm{S} p \mathrm{R} i \mathrm{Ng}$, a difference of $41 \mathrm{msec}$ ). However, any activation of case-sensitive units should increase when the letters are the same size; hence, it is even harder to read pig in spring than in spring, an effect of $63 \mathrm{msec}$.

\section{GENERAL DISCUSSION}

We developed the buried-word task in order to examine the factors that influence attention to subparts of words. We have found the following.

1. Case mixing produced opposite effects on the reading of whole letter strings and on the reading of buried words. Case mixing disrupted the reading of whole strings (see also Besner \& Johnston, 1989) but facilitated the reading of buried words (relative to when the letters were all in the same case; Experiment 1). Items that were most disrupted by case mixing in the whole-word reading test also tended to benefit the most from case mixing in the buried-word task.

2. The slowed naming of words buried in same-case relative to mixed-case strings was not due to interference from responses associated with the complete strings; placing targets within nonword strings had no effect on the effects of case mixing on the naming of buried words (if anything, the data went in the opposite direction; Experiment 2).

3. RTs for the reading of buried words were speeded when target and distractor letters differed in both case and size; these two factors did not interact.

These results provide novel evidence that attention to the subparts of words is affected by letter-based grouping and that this grouping is influenced by case, as well as size, information. Owing to letter-based grouping, buried words are difficult to read when they fall within a string with letters in the same case and size, since the letters making up the buried-word group with the other letters present. Disrupting the grouping, by presenting the target and the distractor letters in different cases and sizes, facilitates performance. There is a positive effect of case mixing on the reading of buried words, exactly the opposite result to that shown when whole strings had to be read (Experiment 1). The beneficial effect of case mixing, for buried words, was not due to response competition from the identity of the whole string, and it was not contingent on grouping based on access to identity information across the whole string; if either of these factors had been important, it should have been more difficult to read buried words in same-case words than in same-case nonwords. There was no evidence for this (Experiment 2). Instead, the data were consistent with grouping's operating at a subword, but supraletter, level.

In Experiment 3, the effects of case and size did not interact in the buried-word task. From this, we may distinguish two forms of grouping. We propose that there is an early grouping process based on size similarity. This 
is followed by a subsequent grouping process contingent upon whether letters activate common visual units. Letters are grouped if they activate units sensitive to their combined occurrence (e.g., at the bigram level). We suggest that, at this second stage, units are influenced by cooccurring letter shapes and, so, respond more strongly to letters in the same case (since they will typically cooccur more often than letters in a different case). Size differences between letters weaken activation of these supraletter visual units, although the units are still activated to some degree by letters presented in the same case. The argument that letters are grouped by activating a common visual unit is supported by neuropsychological evidence. For example, Kumada and Humphreys (2001) tested a patient with extinction of letters in his left visual field when a letter was presented simultaneously in his right field (e.g., there would be a failure to detect the letter $o$ in the stimulus $o g$ ). They found that extinction was reduced when the letters made a word, but only when the letters were also in the same case (in $g o$, but not in $g O$ ). The reduction in extinction can be attributed to grouping between the letters, which enables the left-side letter to be recovered for report. As here, this grouping effect was sensitive to case and size differences between letters.

\section{Implications for Reading?}

In addition to helping us understand the factors that determine attention to the subparts of letter strings, the present data are also relevant to accounts of how case mixing influences whole-word reading. For example, in Experiment 1, we found that the magnitude of the casemixing benefit on buried-word reading was correlated with the magnitude of case-mixing costs on whole-word reading. The processes that contribute to the case effects on buried-word reading thus appear to contribute to case effects on whole-word reading tasks. Within the literature on word recognition, several contrasting accounts have been offered to explain the effects of case mixing on whole-word reading. These include the following: (1) that the effects are due to the masking of lowercase letters by the uppercase letters in mixed-case strings (Besner \& Johnston, 1989), (2) that they are due to the need to analyze more letter forms (Besner \& Johnston, 1989), (3) that case mixing disrupts the recognition of holistic word patterns (Smith, 1969), and (4) that case mixing impairs supraletter grouping (Mayall et al., 1997). The masking proposal is contradicted by our results. Since masking is presumed to affect early stages of letter processing, it should affect buried-word reading in a way similar to that for the reading of whole strings (in particular, the letters in lowercase buried words should be degraded and difficult to read). Evidently, this did not occur. Buried words were easier to read in mixed than in single case, and this held for buried words in lower- as well as in uppercase (there were no interactions of case mixing with the type of form). The number of letter forms and holistic word reading accounts also do not receive positive support from our results. Both of these proposals hold that case mixing should have little effect on the reading of buried words, because (1) the letters making up the buried word were always in the same case (whether embedded within same- or mixed-case strings) and (2) buried words never have a familiar overall shape, due to the presence of the interspersed distractor letters (which break up the holistic shape of the buried word, irrespective of whether the distractor letters are in the same case as or in a different case from the target letters). Our data falsify this null effect prediction. Nevertheless, it could be that an extra factor enters into the buried-word task that plays no part in reading at the whole-string level-namely, attention to the subparts of words. If attention to the buried words is facilitated by case mixing, this may help performance here, but not when the task requires reading at the whole-word level. In contrast to the other proposals, the grouping account does correctly predict that case mixing should have a positive influence on the reading of buried words, since it prevents grouping between target and distractor letters that are the same case and size. It is parsimonious to assume that letter grouping will influence reading at the level of the whole string, as well as the reading of buried words. It follows that letter grouping will play a contributory role in reading and in the negative effects of case mixing shown in whole-word report.

\section{REFERENCES}

BeSNER, D., \& Johnston, J. C. (1989). Reading and the mental lexicon: On the interaction of visual, orthographic, phonological and lexical information. In W. Marslen-Wilson (Ed.), Lexical representation and processes (pp. 291-316). Cambridge, MA: MIT Press.

BESNER, D., \& MCCANN, R. (1987). Word frequency and pattern distortion in visual word identification and production: An examination of four classes of models. In M. Coltheart (Ed.), Attention and performance XII: The psychology of reading (pp. 201-219). Hillsdale, NJ: Erlbaum.

CARR, T. H., \& POllatseK, A. (1985). Recognizing printed words: A look at current models. In D. Besner, T. G. Waller, \& G. E. MacKinnon (Eds.), Reading research: Advances in theory and in practice $V$ (pp. 1-82). Orlando, FL: Academic Press.

CAttell, J. M. (1886). The time taken up by cerebral operations. Mind, 11, 220-242, 377-392.

Coltheart,M., \& Freeman, R. (1974). Case alternation impairs word identification. Bulletin of the Psychonomic Society, 3, 102-104.

DunCAN, J., \& Humphreys, G. W. (1989). Visual search and stimulus similarity. Psychological Review, 96, 433-458.

Frederiksen, J. R., \& Kroll, J. F. (1976). Spelling and sound: Approaches to the internal lexicon. Journal of Experimental Psychology: Human Perception \& Performance, 2, 361-379.

Hodsoll, J., \& Humphreys, G. W. (2001). Driving attention with the top down: The relative contribution of target templates to the linear separability effect in the size dimension. Perception \& Psychophysics, 63, 918-926.

Kumada, T., \& Humphreys, G. W. (2001). Lexical recovery from extinction: Interactions between visual form and stored knowledge modulate visual selection. Cognitive Neuropsychology, 18, 465-478.

LABERGE, D. (1983). Spatial extent of attention to letters and words. Journal of Experimental Psychology: Human Perception \& Performance, 9, 371-379.

MACLEOD, C. M. (1992). The Stroop task: The gold standard of attentional measures. Journal of Experimental Psychology: General, 121, $12-14$. 
Mayall, K. [A.], \& Humphreys, G. W. (1996). Case mixing and the task sensitive disruption of lexical processing. Journal of Experimental Psychology: Learning, Memory, \& Cognition, 22, 278-294.

Mayall, K. A., Humphreys, G. W., \& Olson, A. (1997). Disruption to word or letter processing? The origins of case-mixing effects. Journal of Experimental Psychology: Learning, Memory, \& Cognition, 23, 1275-1286.

MCCANN, R. S., \& BESNER, D. (1987). Reading pseudohomophones: Implications for models of pronunciation assembly and the locus of word frequency effects in naming. Journal of Experimental Psychology: Human Perception \& Performance, 13, 14-24.

MCClelland, J. L., \& Rumelhart, D. E. (1981). An interactive activation model of context effects in letter perception: 1 . An account of basic findings. Psychological Review, 88, 375-407.

Patterson, K. E., \& Coltheart, V. (1987). Phonological processes in reading: A tutorial review. In M. Coltheart (Ed.), Attention and performance XII: The psychology of reading (pp. 421-447). Hillsdale, NJ: Erlbaum.

REICHER, G. M. (1969). Perceptual recognition as a function of meaningfulness of stimulus material. Journal of Experimental Psychology, 81, 274-280.

RENSINK, R. A., \& ENNS, J. T. (1995). Preemption effects in visual search: Evidence for low-level grouping. Psychological Review, 102, $101-130$.

SMith, F. (1969). Familiarity of configuration vs discriminability of features in the visual identification of words. Psychonomic Science, 14, 261-263.

STERNBERG, S. (1969). The discovery of processing stages: Extensions of Donders' method. In W. G. Koster (Ed.), Attention and performance II. Amsterdam: North-Holland. [Acta psychologica, 30, 276315.]
WeEKes, B. (1997). Differential effects of number of letters on word and nonword naming latency. Quarterly Journal of Experimental Psychology, 50A, 439-456.

WheE LER, D. D. (1970). Processes in word recognition. Cognitive Psychology, 1, 59-85.

\section{NOTES}

1. The other side of this argument is that case and size differences may serve as cues to facilitate the selection of the buried words. However, it is likely that grouping and selection are intimately related; selection is facilitated because grouping is decreased between the letters in the buried word and the other letters present. We will present the argument here in terms of grouping, since this can parsimoniously account for data on the reading of mixed-case words, where grouping between letters in the same case and size would be disruptive to performance (Mayall et al., 1997).

2. $F_{1}$ analyses were conducted using participants as a random factor, and $F_{2}$ analyses with letter string as a random factor.

3. When the letters differ in size, the most difficult condition should be that in which the letters in the buried word are small and the distractor letters large, as suggested by results on visual search for small and large targets (e.g., Hodsoll \& Humphreys, 2001). Hence, here we examined the most difficult condition for reading buried words when the letters differed in size, which will be against our hypothesis that size differences facilitate buried-word reading. Note, however, that in Experiment 1 we failed to find any effects of whether the letters in the buried word were small and lowercase versus whether they were large and uppercase.

(Manuscript received November 30, 2001; revision accepted for publication September 24, 2002.) 